\title{
Clinical utility of perfusion (Q)-single-photon emission computed tomography (SPECT)/CT for diagnosing pulmonary embolus (PE) in COVID-19 patients with a moderate to high pre-test probability of PE
}

\author{
Jeeban P. Das ${ }^{1}$ (D) $\cdot$ Randy Yeh ${ }^{1} \cdot$ Heiko Schöder ${ }^{1}$ \\ Received: 23 July 2020 / Accepted: 14 September 2020 / Published online: 22 September 2020 \\ (C) Springer-Verlag GmbH Germany, part of Springer Nature 2020
}

\begin{abstract}
Purpose We reviewed the clinical utility of perfusion (Q)-single-photon emission computed tomography (SPECT)/CT for diagnosing pulmonary embolus (PE) in patients hospitalized with severe acute respiratory syndrome coronavirus 2 (SARSCoV2).

Methods Following the World Health Organization's declaration of a global pandemic, our department policy recommended Qonly SPECT/CT for all patients undergoing nuclear medicine evaluation for suspected PE to reduce the risk of aerosolization of respiratory droplets. We performed a retrospective review of sequential patients admitted with COVID-19 imaged with QSPECT/CT between March 17, 2020, and June 30, 2020, at Memorial Sloan Kettering Cancer Center. We recorded patient demographics, clinical symptoms, Wells score (to stratify patients according to pre-test probability for PE prior to Q-SPECT/CT), and noted ancillary imaging findings on CT.

Results Of the 33 patients imaged with Q-SPECT/CT, 6 patients ( 3 men, 3 women) had a laboratory confirmed diagnosis of COVID-19 (mean age, 55, \pm 11.4 years, range 33-68). All patients had a current diagnosis of malignancy and had a moderate or high pre-test probability for PE (mean Wells score 2.8, range 2-4). Q-SPECT/CT was positive in 4/6 (67\%) of patients. Distribution of pulmonary emboli was bilateral and segmental in $75 \%$ of patients. Ancillary acute findings on SPECT/CT included bilateral parenchymal ground glass opacities $(n=5)$, pleural effusions $(n=2)$, and pneumomediastinum $(n=1)$.

Conclusion Q-SPECT/CT has clinical utility for diagnosing PE in patients with COVID-19 where there is a contraindication for iodinated contrast media and a moderate or high pre-test probability for PE.
\end{abstract}

Keywords COVID-19 $\cdot$ Pulmonary embolism $\cdot$ V/Q SPECT/CT $\cdot$ Coagulopathy

\section{Introduction}

The novel coronavirus disease 2019 (COVID-19), caused by the severe acute respiratory syndrome coronavirus 2 (SARSCOV2) originating in Wuhan, China, in December 2019, has now spread globally and was declared a pandemic by the World Health Organization in March 2020 [1].

This article is part of the Topical Collection on Infection and inflammation

Jeeban P. Das

dasj@mskcc.org

1 Department of Radiology/Nuclear Medicine, Memorial Sloan Kettering Cancer Center, New York, NY, USA
Hospitalized patients most commonly present with mild respiratory symptoms but can undergo rapid clinical deterioration as a result of severe pneumonia requiring intubation and ventilation [2]. Venous thromboembolic (VTE) disease diagnosed in patients with COVID-19 has been identified as a potential cause of accelerated clinical decline, potentiated by endothelial damage and excessive inflammation resulting in a prothrombotic state and disseminated intravascular coagulation [3].

Indeed, the incidence of pulmonary embolus (PE) has been reported in up to $37 \%$ on CT pulmonary angiography (CTPA) in hospitalized COVID-19 patients suspected to have a pulmonary embolus [4]. CTPA remains the most widely used imaging study for diagnosing PE; however, contraindications to iodinated intravenous (IV) contrast including acute kidney 
injury (AKI) and significant allergy to iodine-based contrast agents may preclude select patients suspected to have PE from undergoing CTPA [5]. AKI can develop in up to $37 \%$ of patients admitted to hospital with COVID-19 leaving physicians with the difficult decision of deciding whether the benefit of the imaging study to rule out PE outweighs the risk of administering IV contrast [6].

Ventilation (V)-Perfusion (Q) SPECT/CT has been validated as both safe and acceptable for evaluating PE and can accurately establish the diagnosis even in the presence of chronic cardiopulmonary disease or pneumonia as well as having no contraindications [7, 8]. Nevertheless, the risk of nosocomial infection and healthcare worker exposure as a result of the potential aerosolization of SARS-CoV2 virus particles during the imaging acquisition of the ventilation component of V/Q scanning poses cause for concern. In addition, poor respiratory effort in COVID-19 patients may cause inhaled particles to localize to the trachea and central bronchi and not demonstrate alveolar ventilation. Satisfactory disinfection of the ventilation system following ventilation scanning during the COVID-19 pandemic may also be prohibitively time consuming in a high-volume nuclear medicine department [9-11].

Since the rapid emergence of the COVID-19 pandemic, diagnostic algorithms for the evaluation of PE which minimize the performance of ventilation studies have been proposed, limiting the potential for aerosolization and exposure of COVID-19 to patients and staff members [10-13]. However, prior societal guidelines have suggested the use of both perfusion and ventilation scans in patients suspected to have PE to reduce the potential risk of false positive results $[8$, 14]. The addition of a low-dose CT to SPECT perfusion imaging has been shown to mitigate this potential risk and improve the specificity of the perfusion exam [7].

The objective of our study was to demonstrate the clinical utility of perfusion only (Q) SPECT/CT imaging in the diagnosis of PE in COVID-19 patients with a moderate to high pre-test probability for $\mathrm{PE}$.

\section{Materials and methods}

\section{Patient selection}

This retrospective study received approval from the Memorial Sloan Kettering Cancer Center Institutional Review Board (IRB\#20-194). The requirement for individual patient consent was waived. All patients referred for Q-only SPECT/CT to assess for PE between March 2020 and June 2020 were reviewed. Patients with a laboratory-confirmed diagnosis of COVID-19 who underwent Q-SPECT/CT were included in our final analysis. All patients had a positive real-time polymerase chain reaction COVID-19 nasopharyngeal swab test.
Patient demographics, smoking history, and Wells score were recorded.

\section{Imaging protocols and acquisition}

Imaging data were recorded with the Precedence 6 (Philips Healthcare, Best, The Netherlands) or Symbia T2 (Siemens Medical Solution Inc., IL, USA) SPECT/CT scanning systems. Low-dose CT scans were recorded at $120 \mathrm{kVp}, 15$ to $30 \mathrm{mAs}$. No IV contrast was administered. Following CT acquisition, SPECT images of the chest were recorded for $20 \mathrm{~s} / \mathrm{step}$ in a standard $128 \times 128$ matrix. Q-SPECT imaging data were reconstructed, fused with the corresponding CT image slices, and were displayed in axial, coronal, and sagittal planes.

\section{Image interpretation}

Images were analyzed using 3D viewing software suite (Hermes Hybrid Viewer, Hermes Medical Solutions). The planar and SPECT/CT scan datasets were interpreted clinically on separate occasions at time of imaging acquisition and rereviewed for the purpose of this manuscript using previously published criteria. PE was suspected on Q-SPECT/CT scans when $\geq 1$ wedge-shaped peripheral defect estimated as greater than or equal to $50 \%$ of a pulmonary segment identified in all three orthogonal planes without a corresponding abnormality on CT [7].

\section{Results}

Patient selection is shown in Fig. 1. In brief, during the study period, 33 patients underwent Q-SPECT/CT imaging. Of these patients, six had a laboratory-confirmed diagnosis of COVID-19. All patients had a current diagnosis of malignancy. Two patients had a smoking history. No patient had a history of reactive airway disease or asthma. Patient demographics and clinical features are outlined in Table 1.

Five of six patients had acute kidney injury (AKI) or acute on chronic kidney injury with a serum creatinine $\geq$ $133 \mu \mathrm{mol} / \mathrm{L}$. One patient had a history of severe allergic reaction to iodinated contrast media. Two patients underwent lower limb Doppler ultrasonography prior to Q-SPECT/CT demonstrating no evidence for deep vein thrombosis. Q-SPECT/ CT was positive in 4/6 (67\%) of patients. One patient had a diagnosis of a segmental left upper lobe pulmonary embolus on CT pulmonary angiography study performed 2 weeks prior to Q-SPECT/CT imaging (subsequently performed for worsening dyspnea in the context of $\mathrm{AKI}$ ) re-demonstrating the segmental PE (Fig. 2). The distribution of pulmonary emboli was bilateral and segmental in 3/4 (75\%) of COVID-19 patients with a positive Q-SPECT/CT study (Fig. 3). In the non- 
Table 1 Patient demographics of laboratory-proven COVID-19 patients imaged with Q-SPECT/CT

\begin{tabular}{|c|c|c|c|c|c|c|}
\hline Patient cohort & Patient 1 & Patient 2 & Patient 3 & Patient 4 & Patient 5 & Patient 6 \\
\hline Age (years) & 68 & 59 & 70 & 71 & 31 & 33 \\
\hline Gender & Male & Female & Female & Female & Male & Male \\
\hline Malignancy & Leukemia & Breast & Leukemia & Myeloma & Testicular & Gastric \\
\hline Clinical presentation & Dyspnea & Fever & Dyspnea & Dyspnea & Fever & Chest pain \\
\hline D-dimers $(\mu \mathrm{g} / \mathrm{L})$ & 14,700 & 9100 & 2200 & 1700 & Not performed & Not performed \\
\hline Serum creatinine $(\mu \mathrm{mol} / \mathrm{L})$ & 212 & 142 & 133 & 548 & Normal & 133 \\
\hline Wells score & 2 & 3 & 3 & 4 & 2 & 3 \\
\hline Hospital admission to Q-SPECT/CT (days) & 19 & 12 & 11 & 15 & 4 & 8 \\
\hline Positive study & Yes & Yes & No & Yes & No & Yes \\
\hline Treatment & Anticoagulation & Anticoagulation & $\mathrm{n} / \mathrm{a}$ & Anticoagulation & $\mathrm{n} / \mathrm{a}$ & Anticoagulation \\
\hline ICU admission & Yes & No & No & No & No & Yes \\
\hline
\end{tabular}

COVID patient cohort who underwent Q-SPECT/CT, 4/27 $(15 \%)$ had a positive diagnosis of PE. No Q-SPECT/CT imaging study was considered non-diagnostic in either the nonCOVID or COVID-positive patient groups.

Classic features of COVID-19 pneumonia were seen in five patients, including bilateral GGOs and consolidative opacities. Additional acute findings on chest CT were seen in three patients including pleural effusions $(n=2)$ and pneumomediastinum $(n=1)$. Non-acute $\mathrm{CT}$ findings included thoracic adenopathy $(n=2)$, pulmonary metastasis $(n=1)$, and pulmonary fibrosis $(n=1)$. The low-dose CT scan was entirely normal in only $1 / 6$ patients $(18 \%)$.

The median time from hospital admission to Q-SPECT/CT imaging was 11.4 days (range 3-19 days). Of the six patients hospitalized with COVID-19, two were admitted to the Intensive Care Unit for mechanical ventilation following the diagnosis of pulmonary embolus and eventually expired. The mean duration of hospitalization was 40.5 days (range 661 days).

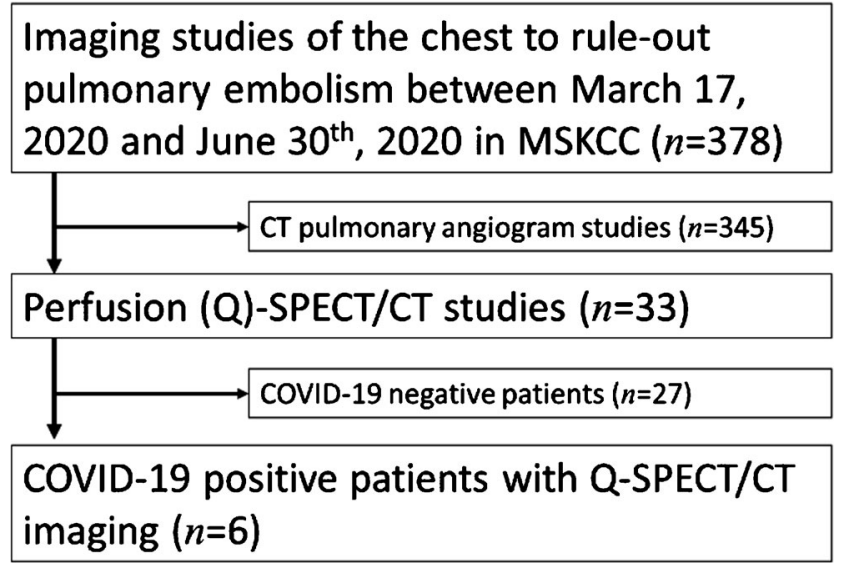

Fig. 1 Flowchart showing the patients included in our final analysis

\section{Discussion}

The role of Q-SPECT/CT in COVID patients has been raised in several clinical editorials to date $[11,15-17]$. We describe the clinical utility of Q-only SPECT/CT in diagnosing PE in COVID-19-positive patients with a contraindication to iodinated contrast media and a moderate-to-high risk of PE. In our cohort, $67 \%$ had a positive diagnosis of PE on Q-SPECT/CT. Classical features of COVID-19 pneumonia were seen in 5 patients and rare manifestations seen in 3 patients (pleural effusions $n=2$, pneumomediastinum $n=1$ ).

CT imaging of the thorax in patients with COVID-19 plays a crucial role in patient evaluation in the both the diagnosis of COVID-19 pneumonia and in evaluating thromboembolic events $[2,18]$. However, the clinical usefulness of QSPECT/CT in hospitalized COVID-19 patients with moderate to high risk of PE has yet to be evaluated. We used the Wells score, a clinically validated scoring system to evaluate for the presence of a venous thromboembolic event (VTE). A Wells score of $\leq 1$ can reliably rule out the possibility of VTE in select patients with a score of 2 denoting a moderate risk and $\geq 3$ a high risk [19]. We have demonstrated that the combination of Q-SPECT with low-dose CT is a useful modality to diagnose VTE in COVID-19 patients clinically suspected to have PE.

In routine V/Q SPECT imaging studies, the ventilation component may improve the specificity of the perfusion study and there is already data showing that the addition of low-dose CT to the SPECT images, the corroborated reporting of the scans with the structural information leads to a higher sensitivity, comparable with that of CTPA [20, 21]. Cobes et al. retrospectively analyzed V/Q SPECT/CTs in 5 patients hospitalized with COVID-19 and determined that V/Q SPECT/ CT could play a role diagnosing embolic complications while following meticulous hygiene [22]. However, combining a low-dose chest CT with Q-only SPECT can also satisfactorily 
Fig. 2 Axial fused perfusion SPECT and CT (a) and axial perfusion SPECT (b)

demonstrating a left upper lobe wedge-shaped defect (arrows) suspicious for pulmonary embolism. Axial CT chest images showing ancillary findings of pneumomediastinum (arrow) (c) and bibasal subpleural airspace disease (arrows) (d) suspicious for COVID-19 pneumonia

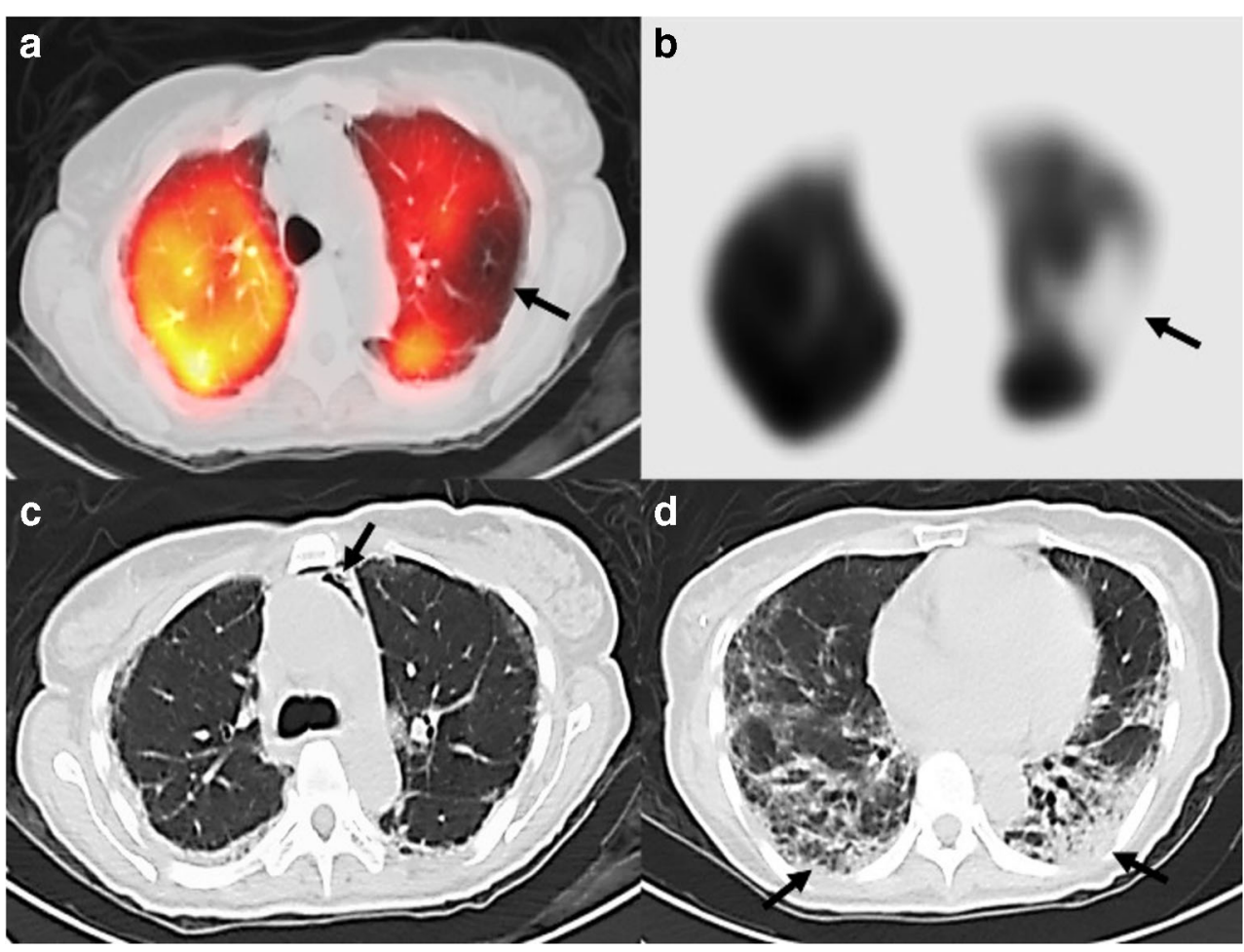

demonstrate etiologies of potential non-embolic false positive results, such as pulmonary emphysema or bullous disease [23]. Ventilation studies can contaminate staff members and equipment and in a high-volume nuclear medicine institution, the necessary decontamination steps may not be offset by the benefit of ventilation imaging in some patients during the
Fig. 3 Axial fused Q-SPECT/CT (a) and SPECT (b) showing a left upper lobe wedge-shaped defect (arrows) suspicious for pulmonary embolism. An additional right lower lobe wedge-shaped defect was also seen (not shown). Axial CT chest images $(\mathbf{c}, \mathbf{d})$ showing small bilateral pleural effusion and bibasilar GGOs suspicious for COVID-19 pneumonia

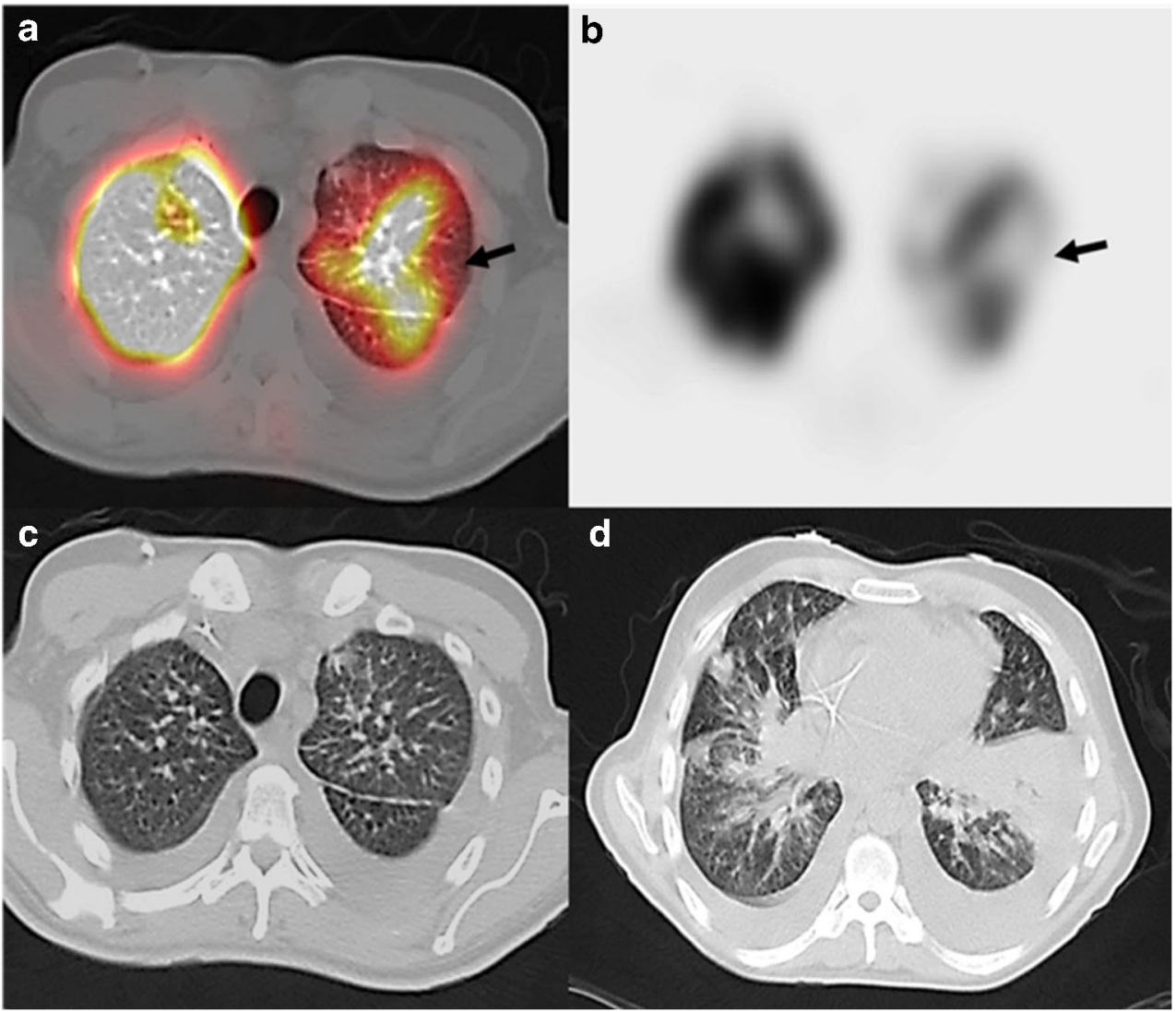


COVID-19 pandemic. In addition, performing ventilation scans in clinically compromised COVID-19 patients may be both technically challenging and time consuming and may greatly increase the risk of viral transmission [24].

Diagnosing PE on Q-SPECT/CT has been validated in several previous studies. Lu et al. demonstrated that QSPECT/CT had a higher accuracy than planar V/Q imaging to diagnose $\mathrm{PE}$ in patients with a high pre-test probability for PE [7]. Mazurek et al. performed a retrospective review of 84 patients to evaluate the utility of Q-SPECT/CT in the diagnosis of PE. The authors reported a similar sensitivity and specificity to $\mathrm{Lu}$ et al. (100 and $83 \%$, respectively) concluding that Q-SPECT/CT imaging had a high diagnostic efficacy in evaluating patients suspected to have PE. In addition, the inclusion of a chest CT (even at low dose) adds value by characterizing acute pulmonary pathology which could account for the patient's clinical presentation [23]. In addition, based on the current European Association of Nuclear Medicine guidelines, Q-only SPECT is already recommended as standard of care, for example in pregnant patients with suspected PE [8].

Several prior studies have described the frequently seen COVID-19 imaging findings on CT chest. The most common imaging features include multifocal bilateral peripheral GGOs and subsegmental and subpleural patchy consolidations predominating in the lower lung lobes [2]. The image quality of low-dose CT studies can be considered comparable with that of higher dose CT for the detection of gross mediastinal and parenchymal lung changes $[25,26]$. Of the five patients with acute findings on the low-dose CT chest, all demonstrated these classical features of COVID-19 pneumonia. Three patients showed rare findings associated with COVID-19. Pleural effusions, considered a rare feature of COVID-19, were seen in $33 \%$ of our patient cohort but are typically seen in only $1-3 \%$ of reported hospitalized COVID-19 cases [2, 12]. This may be explained by our unique patient population, all with a diagnosis of active malignancy at time of imaging. Of the two patients with pleural effusions, both had thoracic nodal or pulmonary metastases. Pneumomediastinum (seen in one patient) has thus far only been described in case reports of patients with COVID-19 but may indicate a further potential clinical decline and is an important ancillary finding on companion chest CT of Q-only SPECT/CT [27, 28].

We note several limitations of our study. In the first instance, we are limited by our small sample size and the retrospective nature of our analysis. Secondly, selection bias may have impacted the decision to pursue Q-SPECT/CT as an imaging modality for certain patients with COVID19 who experienced a clinical decline during hospitalization. Thirdly, our entire patient population was derived from a single cancer-specialist center and all had an active malignancy at time of imaging limiting generalizability to other hospital populations.
In conclusion, Q-SPECT/CT is a reasonable first choice imaging modality for diagnosing PE in COVID-19 patients with a medium to high probability of PE.

Data availability Data is available upon request.

\section{Compliance with ethical standards}

Conflict of interest The authors declare that they have no conflict of interest.

Ethics approval Informed consent was obtained. This study was approved by the Memorial Sloan Kettering Cancer Center Institutional Review Board (IRB\#20-194).

\section{References}

1. Zhou F, Yu T, Du R, Fan G, Liu Y, Liu Z, et al. Clinical course and risk factors for mortality of adult inpatients with COVID-19 in Wuhan, China : a retrospective cohort study. Lancet. 2020;673(6):1-9.

2. Simpson S, Kay FU, Abbara S, Bhalla S, Chung JH, Chung M, et al. Radiological Society of North America expert consensus statement on reporting chest CT findings related to COVID-19. Endorsed by the Society of Thoracic Radiology, the American College of Radiology, and RSNA - Secondary Publication. J Thorac Imaging 2020.35:219-227.

3. Poissy J, Goutay J, Caplan M, Parmentier E, Duburcq T, Lassalle F, et al. Pulmonary embolism in COVID-19 patients: awareness of an increased prevalence. Circulation. 2020;142:184-6.

4. Kaminetzky M, Moore W, Fansiwala K, Babb JS, Kaminetzky D, Horwitz LI. Pulmonary embolism on CTPA in COVID-19 patients. Radiol Cardiothoracic Imag. 2020;2.4:e200308.

5. Konstantinides SV, Torbicki A, Agnelli G, et al. ESC guidelines on the diagnosis and management of acute pulmonary embolism. Eur Heart J. 2014;35:3033-3069k.

6. Hirsch JS, Ng JH, Ross DW, Sharma P, Shah HH, Barnett RL, et al. Acute kidney injury in patients hospitalized with COVID-19. Kidney Int. 2020:98.209-18.

7. Lu Y, Lorenzoni A, Fox JJ, Rademaker J, Vander Els N, Grewal RK, et al. Noncontrast perfusion single-photon emission CT/CT scanning: a new test for the expedited, high-accuracy diagnosis of acute pulmonary embolism. Chest. 2014;145:1079-88.

8. Bajc $\mathrm{M}$, Schümichen $\mathrm{C}$, Grüning $\mathrm{T}$, et al. EANM guideline for ventilation/perfusion single-photon emission computed tomography (SPECT) for diagnosis of pulmonary embolism and beyond. Eur J Nucl Med Mol Imaging. 2019;46:2429-51.

9. Seto WH, Tsang D, Yung RW, Ching TY, Ng TK, Ho M, et al. Effectiveness of precautions against droplets and contact in prevention of nosocomial transmission of severe acute respiratory syndrome (SARS). Lancet. 2003;361:1519-20.

10. Zuckier LS, Moadel RM, Haramati LB, Freeman LM. Diagnostic evaluation of pulmonary embolism during the COVID-19 pandemic. J Nucl Med. 2020;61:630-1.

11. Burger IA, Niemann T, Patriki D, Fontana F, Beer JH. Is there a role for lung perfusion $\left[{ }^{99 \mathrm{~m}} \mathrm{Tc}\right]-\mathrm{MAA}$ SPECT/CT to rule out pulmonary embolism in COVID-19 patients with contraindications for iodine contrast? Eur J Nucl Med Mol Imaging. 2020;47:2062-3.

12. Lu Y, Macapinlac HA. Perfusion SPECT / CT to diagnose pulmonary embolism during COVID-19 pandemic inflammation, vol. 8; 2020. p. 1-2. 
13. Kooraki S, Hosseiny M, Myers L, Gholamrezanezhad A. Coronavirus outbreak : what the department of radiology should know acknowledgments. J Am Coll Radiol. 2020, 2020:17.44751.

14. Bajc M, Neilly JB, Miniati M, Schuemichen C, Meignan M, Jonson B. EANM guidelines for ventilation / perfusion scintigraphy. Eur J Nucl Med Mol Imaging. 2009;36:1528-38.

15. Vöö S, Neriman D, Henry M, Kayani I. Perfusion-ventilation imaging in an era of COVID-19. Nucl Med Commun. 2020;41(6): 597-8. https://doi.org/10.1097/MNM.0000000000001201.

16. Burger IA, Niemann T, Patriki D, Fontana F, Beer JH. Lung perfusion [ $\left.{ }^{99 \mathrm{~m}} \mathrm{Tc}\right]-M A A$ SPECT/CT to rule out pulmonary embolism in COVID-19 patients with contraindications for iodine contrast. Eur J Nucl Med Mol Imaging. 2020;47:2209-10.

17. Vöö S, Dizdarevic S. Single photon emission computed tomography lung perfusion imaging during the COVID-19 pandemic: does nuclear medicine need to reconsider its guidelines? Nucl Med Commun. 2020;41:991-3.

18. Bernheim A, Mei X, Huang M, Yang Y, Fayad ZA, Zhang N, et al. Chest CT findings in coronavirus disease-19 (COVID-19): relationship to duration of infection. Radiology. 2020;295:200463.

19. Modi S, Deisler R, Gozel K, Reicks P, Irwin E, Brunsvold M, et al. Wells criteria for DVT is a reliable clinical tool to assess the risk of deep venous thrombosis in trauma patients. World J Emerg Surg. 2016;11:24.

20. Bhatia KD, Ambati C, Dhaliwal R, et al. SPECT-CT/VQ versus CTPA for diagnosing pulmonary embolus and other lung pathology: pre-existing lung disease should not be a contraindication. J Med Imaging Radiat Oncol. 2016;60:492-7.
21. Miniati M, Sostman HD, Gottschalk A, Monti S, Pistolesi M. Perfusion lung scintigraphy for the diagnosis of pulmonary embolism: a reappraisal and review of the prospective investigative study of acute pulmonary embolism diagnosis methods. Semin Nucl Med. 2008;38:450-61.

22. Cobes N, Guernou M, Lussato D, Queneau M, Songy B, Bonardel $\mathrm{G}$, et al. Ventilation / perfusion SPECT / CT findings in different lung lesions associated with COVID-19 : a case series. Eur J Nucl Med Mol Imaging. 2020;18:1-8.

23. Mazurek A, Dziuk M, Witkowska-Patena E, Stanislaw P, Gizewska A. The utility of hybrid SPECT / CT lung perfusion scintigraphy in pulmonary embolism diagnosis. Respiration. 2015;90:393-401.

24. Williams DA, Carlson C, Mcenerney K, Hope E, Hob CK. Technetium-99m DTPA aerosol contamination in lung ventilation studies. J Nucl Med Technol. 1998;26:43-5.

25. Ravenel JG, Scalzetti EM, Huda W, Garrisi W. Radiation exposure and image quality in chest CT examinations. AJR Am J Roentgenol. 2001;177:279-84.

26. Karabulut N, Törü $M$, Gelebek V, Gülsün $M$, Ariyürek OM. Comparison of low-dose and standard-dose helical CT in the evaluation of pulmonary nodules. Eur Radiol. 2002;12(11):2764-9.

27. Zhou C, Gao C, Xie Y, Xu M. COVID-19 with spontaneous pneumomediastinum. Lancet Infect Dis. 2020;20:510.

28. Romano N, Fischetti A, Melani EF. Pneumomediastinum related to Covid-19 pneumonia [published online ahead of print, 2020 Jun 6]. Am J Med Sci. 2020;S0002-9629(20)30245-7.

Publisher's note Springer Nature remains neutral with regard to jurisdictional claims in published maps and institutional affiliations. 